\title{
Achieving of Magnus Effect with Agros Suite
}

\author{
Aleksandr Lukin ${ }^{1 *}$, Galina Demidova', Anton Rassõlkin ${ }^{1,2}$ \\ ${ }^{1}$ Faculty of Control Systems and Robotics, ITMO University, 197101 St. Petersburg, 49 Kronverksky Pr., Russia \\ 2 Department of Electrical Power Engineering and Mechatronics, School of Engineering, Tallinn University of Technology, \\ 19086 Tallinn, 5 Ehitajate tee, Estonia \\ * Corresponding author, e-mail: alevlukin@itmo.ru
}

Received: 24 December 2020, Accepted: 17 February 2021, Published online: 15 April 2021

\begin{abstract}
When the rotating body gets into the ambiance flow appears the lifting force, called Magnus Effect. That lifting force may be controlled by changing the revolution speed of the body. That phenomenon uses in many engineering applications like wind turbines and marine ships. In this paper, the Magnus Effect simulation is achieved with Agros Suite, a multiplatform application for the solution of physical problems. The article presents the nature of the Magnus Effect and discusses possible applications in engineering. The research question is focused on demonstrating the Magnus Effect with Agros Suite and evaluating the computational power of the personal computer that runs the simulation. The simulation is made keeping in mind the possible application of the Agros Suite tools for Magnus-Effect-based wind generator control algorithms optimization. The simulation result analysis shows that Agros Suite is a reliable tool in accessing and simulation of such phenomena.
\end{abstract}

\section{Keywords}

physics computing, numerical simulation, software, optimization tools

\section{Introduction}

The Magnus Effect is a lifting force produced when a rotating body (in the current case cylinder) produces a pressure ambiance differential. The rotating body's path is deflected in a manner that is not present when the body is not rotating. This is the same force that makes a golf ball or a baseball curve slice. The Magnus Effect is a function of the rotational speed. According to Bernoulli's principle, increasing the speed of an ambiance decreases the pressure [1]. The nature of the Magnus Effect is given in Fig. 1.

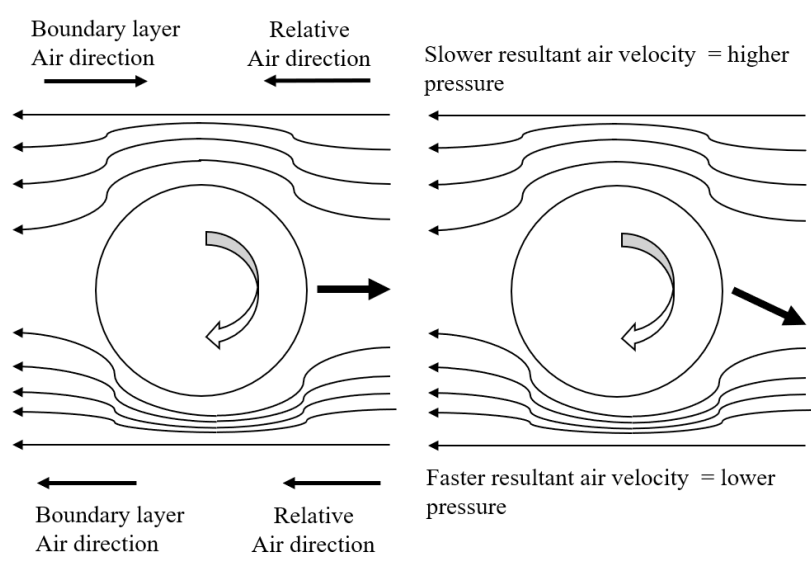

Fig. 1 Nature of Magnus Effect
The first experiment in the field was performed by a German physicist H.G. Magnus in 1853. The scientist used a rapidly rotating brass cylinder and observed significant lateral displacement caused by wind. At the beginning of the $20^{\text {th }}$ century, the Kutta-Joukowski theorem was developed, which allowed estimating forces affecting cylindrical rotor in uniform frictionless airflow. The lift of a cylinder presented as a vortex can be calculated according to Eq. (1).

$L=\rho \cdot V \cdot G$,

where $L$ is the lift of a cylinder per unit length, $V$ is flow velocity, $\rho$ is the density of a fluid, and $G$ is the strength of a vortex. The strength of a vortex $G$ is given by Eq. (2).

$G=2 \cdot \pi \cdot r^{2} \cdot \omega$

where $r$ is the radius of a cylinder and $\omega$ is the angular velocity of the vortex.

This simplified model is often used to describe the Magnus Effect; however, it is based on the frictionless flow and does not take viscosity into account. Another more accurate model is based on the concept boundary layer presented by Ericsson [2]. It explains the Magnus Effect through asymmetrical flow patterns, created by the 
difference in boundary layer separation between the upper and the lower surface of the cylinder, and a phenomenon called the Moving Wall Effect.

According to Seifert [3], the first application of Magnus force in engineering was proposed by Anton Flettner in the 1920s. He conducted research using a model boat with cylindrical rotors installed in place of conventional sails and discovered that the rotor produced significantly higher thrust while having the same equivalent area. This type of vessel propulsion system was called the Flettner rotor (also called turbosail). Two experimental $15 \mathrm{~m}$ high Flettner rotors were later installed on a 2000-ton schooner Buckau, replacing its original sail rig. They were immediately proven to have several significant advantages over conventional sails. The propulsion system's weight was only $1 / 5$ of conventional sails and rigging, while the ship was able to reach the speed of 8 knots compared to 6.5 knots before modification. Using rotor sails also reduced the risk of capsizing and increased the ship's stability during stormy weather. However, despite initial success, two factors prevented the new propulsion system from going mainstream: The Great Depression and marine diesel technology development. Several experiments with planes retrofitted with Magnus rotors in place of fixed wings were conducted. However, those have proven to be inefficient and dangerous due to the inability to glide in the rotor shutdown case.

At the end of the 20th century, ecological concerns and rising oil prices raised interest in new marine propulsion systems. Technological advancements allowed re-evaluating a half-century-old Flettner rotor and creating a new generation of rotor sails to increase fuel economy significantly. This technology is currently used on several commercial vessels, such as E-Ship 1 [4]. This ship uses four Flettner rotors in combination with conventional thrusters, which, according to reports, helps to achieve a $15 \%$ fuel economy. The other successful example of a rotor ship is Viking Grace [5, 6]. Its main component is a single 24-meter-tall cylindrical rotor, which is used as a part of a hybrid propulsion system combined with propellers, saving 300 tons of liquid gas fuel per year.

Another application of the Magnus Effect is alternative energy applications $[7,8]$. Using rotors instead of propeller blades in wind generators potentially hold multiple advantages and attracts growing interest from researchers worldwide [9]. Some of those are:

- A wider range of wind velocities: from 2 to $40 \mathrm{~m} / \mathrm{s}$ compared to $5-25 \mathrm{~m} / \mathrm{s}$ when using bladed turbines.

- High ecological and operational safety. Such turbines are operating with lower rotational speed and do not produce infrasound.

- Thus, the ability to operate with a wide range of weather conditions makes Magnus-type turbines suitable for high and low wind potential areas and makes them a viable alternative to propeller turbines. Some examples of such turbines are presented in Section 2.

In this paper, the Magnus Effect is achieved with Agros Suite [10], a multiplatform application for physical problems based on the Hermes and deal.II library. One of the most useful frameworks of Agros Suite is the Ārtap [11, 12]. $\bar{A}$ rtap framework provides a set of numerical solvers and optimization tools for robust design optimization of electrical machines $[13,14]$.

One of the main advantages of selected software is the introduction of $h, p$, and $h p$-adaptivity. $h$-adaptivity is based on mesh refinement, while the $p$-adaptive method is aimed at changing the degree of approximating polynomial in the given element [15]. $h p$-adaptivity presents a hybrid approach that simultaneously refines both mesh and polynomials. Research shows that $h p$-adaptivity significantly reduces the computational complexity compared to $h$-adaptive techniques [16].

Another approach to simulation of the Magnus effect is the application of CFD software, such as ANSYS CFX. Research shows that this software allows to simulate and observe the Manus effect. The authors also recommend mesh settings for future research [17]. Introduction of CFD software into a chosen field of research allows to perform optimization of cylinders and search for preferred shapes without complex experiments in the wind turbine, as existing papers show [18].

The current work is related to developing an experimental prototype of the high-efficiency wind turbine based on the Magnus Effect [1, 9]. Ārtap framework seems to be a perfect tool for the prototype optimization phase to reach a robust optimum. It provides a simple user interface with integrated optimization and numerical libraries. Ārtap framework has a plain three-layered architecture, where the user needs to define the problem class and rewrite the optimization function.

The main goal of this work is to assess the possibility of simulating the Magnus effect in Agros 2D. To reach this goal, the following tasks were established:

- Describe Magnus cylinder mathematically in terms of rotational speed.

- Design a wind tunnel with pre-determined parameters. 
- Perform a simulation of the Magnus effect at different flow velocities and rotational speeds.

- Perform a visual and numerical analysis of obtained results.

- Assess the applicability of selected software for further research in the field of Magnus effect simulation.

\section{Agros implementation}

\subsection{Mathematical description of the Magnus Effect}

To determine the speed of a cylinder, Agros requires an input of velocities along both horizontal and vertical axes. Solving this task requires analyzing the peripheral speed of the point of the surface of the cylinder. The formula for peripheral speed is Eq. (3), where $r$ is the cylinder's radius, $n$ is the rotational frequency in $1 / \mathrm{s}$, and $d$ is the radius of a cylinder.

$$
U=2 \cdot \pi \cdot r \cdot n=\pi \cdot d \cdot n
$$

To project the peripheral speed on the coordinate axes, Eqs. (4) and (5) were used, where $x_{0}$ is the position of the center of the cylinder on the horizontal axis.

$$
\begin{aligned}
& U_{x}=\pi \times d \times n \times \cos \left(\frac{x-x_{0}}{x_{0}}\right) \\
& U_{y}=\pi \cdot d \cdot n \cdot \sin \left(\frac{x-x_{0}}{x_{0}}\right)
\end{aligned}
$$

In the presented model, both formulas can be described as Eqs. (6) and (7). In cases of these formulas, $n$ can be varied for different rotational speeds.

$$
\begin{aligned}
& U_{x}=\pi \cdot 0.1 \cdot n \cdot \cos \left(\frac{x-0.2}{0.2}\right) \\
& U_{y}=\pi \cdot 0.1 \cdot n \cdot \sin \left(\frac{x-0.2}{0.2}\right)
\end{aligned}
$$

\subsection{Obtaining of Magnus Effect in Agros}

This work aims to perform a simulation of the Magnus Effect using the Agros 2D software. Agros allows running computational analysis to solve problems in different fields, including fluid flow. The user interface of Agros is shown on Fig. 2.

Using the Agros suite, a model of a wind tunnel was built. The length of a tunnel is $2 \mathrm{~m}$, and the diameter is $0.4 \mathrm{~m}$. The diameter of a cylinder is $0.1 \mathrm{~m}$. The designed wind tunnel is presented in Fig. 3.

The space inside the tunnel is filled with air. Properties of gas are presented in Table 1 .
Mesh parameters were adjusted to create more sufficient elements near the boundary area. A triangular mesh was used with polynomial order of 2 and 2 refinements. The mesh is presented in Fig. 4. The final number of elements of the mesh is 8480 . The solver was set to Newton's method.

\section{Results}

Two series of simulations were run, in both cases, the wind speed remained the same, and the cylinder's rotational speed was varied from 0 to $60 \mathrm{~Hz}$ with $5 \mathrm{~Hz}$ step. Each simulation covered a $1 \mathrm{~s}$ period of working of a cylinder, which includes 1000 time steps. The Magnus Effect is based on the difference in flow separation between lower and upper parts of the cylinder, and thus, to evaluate the Effect, two control points were set. One issue is fixed at the cylinder's upper surface, while the other is located at the lower part of the surface. The difference between these two values shows how the flow separation is affected by the flow parameters and the cylinder's parameters. Later the difference between these values was logarithmically approximated and plotted. The graph is shown in Fig. 5.

In the case of $v=8$, three distinct areas can be seen on the plot. The first area, between $0-10 \mathrm{~Hz}$, shows are where the Magnus Effect is insignificant, and the flow velocity doesn't change with an increase in rotational speed. The second area, 10-30 Hz, shows the normal operating area of the Magnus cylinder. In this area, the dependence between flow separation parameter and rotational frequency is quasilinear. Thus, the Magnus-Effect Wind Generator (MEWG) can be controlled by adjusting the speed of cylinders. The third area, 30-60, shows the saturation points at which flow separation becomes unstable. In the last area, the increase in rotational speed leads to a decrease in the difference between the speed rate on the lower and upper parts of the cylinder. This area shows rotational frequencies at which the cylinder becomes ineffective.

Similar processes can be seen at flow velocity $V=12$. The main difference is in the length of the normal operational area. At higher wind speed, it widens from 10-30 to $10-40 \mathrm{~Hz}$. At the saturation point $(40 \mathrm{~Hz})$, the difference between wind speeds is higher than at the saturation point for $V=8(30 \mathrm{~Hz})$. Thus, it can be seen that at higher flow velocities, the width of an area of regular operational frequencies of the Magnus cylinder increases along with flow separation.

A visual analysis of the results of the simulation was also performed. The image was captured for a wind speed of $12 \mathrm{~m} / \mathrm{s}$ and a rotational frequency of $50 \mathrm{~Hz}$. The velocity map is presented in Fig. 6. During the simulation, the cylinder rotated counterclockwise. Thus, the decrease 


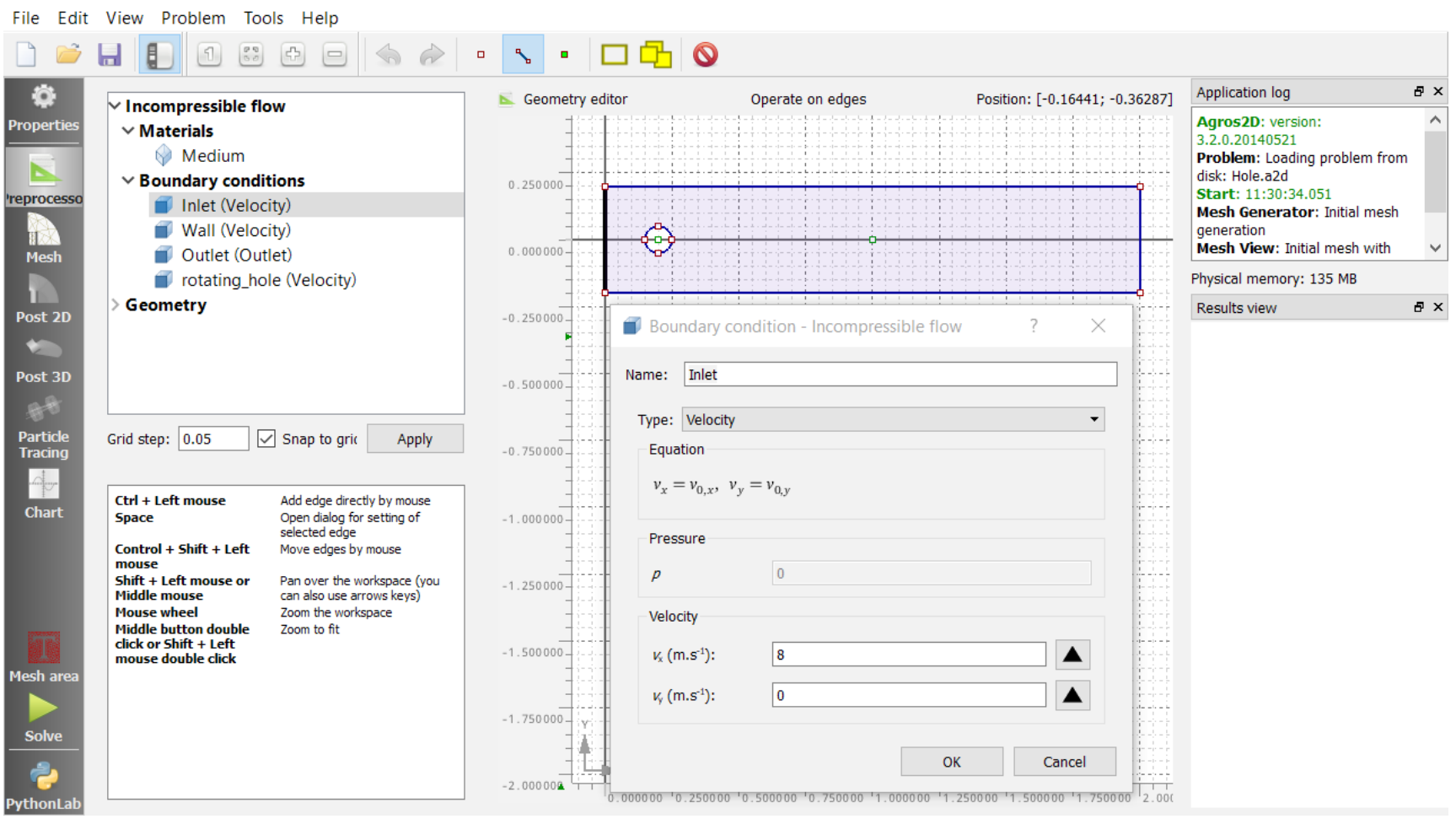

Fig. 2 Screenshot of Agros user interface

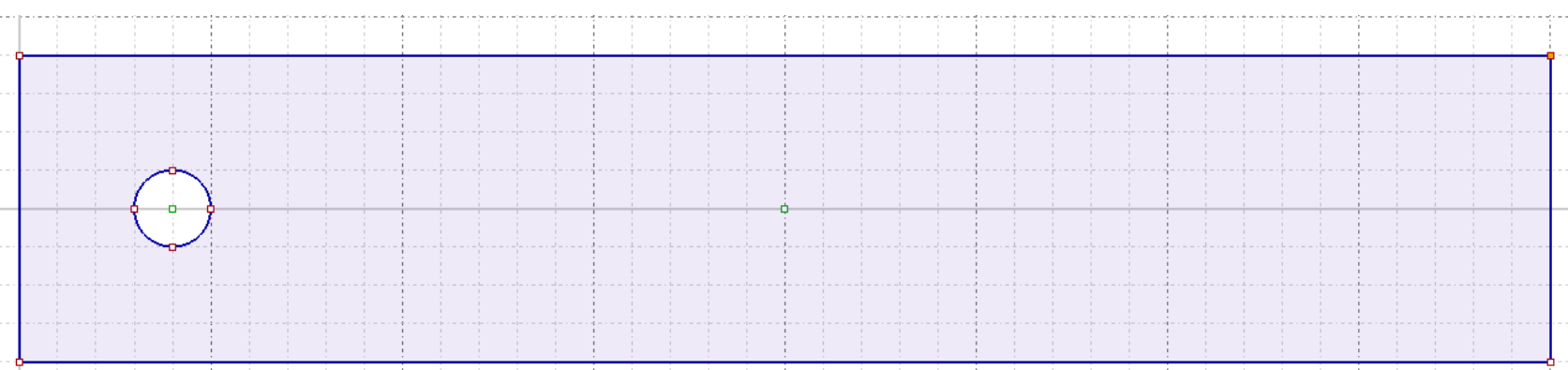

Fig. 3 Wind tunnel sketch in Agros

Table 1 Properties of the gas used in the simulation.

\begin{tabular}{lc}
\multicolumn{2}{c}{ simulation. } \\
\hline Property & Value \\
\hline Density, $\mathrm{kg} / \mathrm{m}^{3}$ & 1 \\
Viscosity, $\mathrm{Pa}_{\mathrm{s}}$ & 0.00018 \\
\hline
\end{tabular}

Fig. 4 Mesh of wind tunnel in Agros 


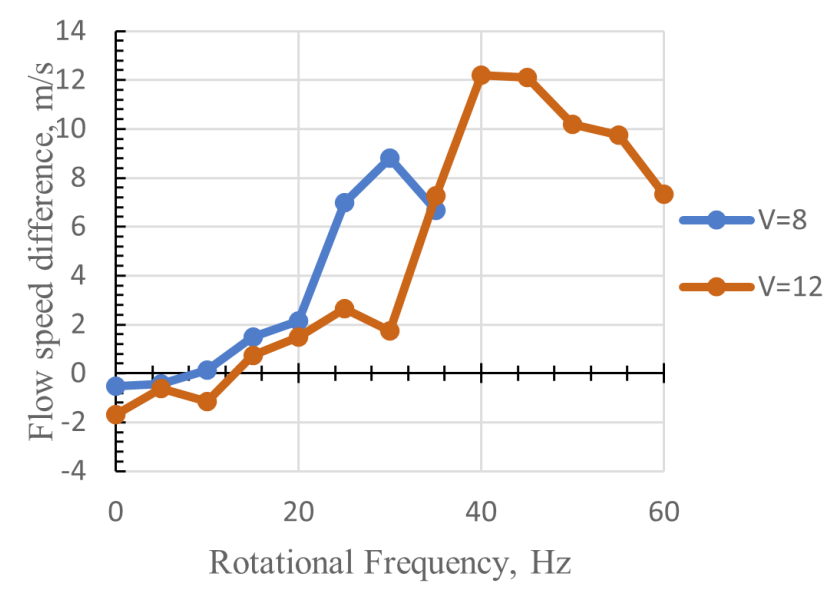

Fig. 5 Difference between velocity in two control points compared to the rotational speed of the cylinder for different wind speeds $(\mathrm{V}, \mathrm{m} / \mathrm{s})$

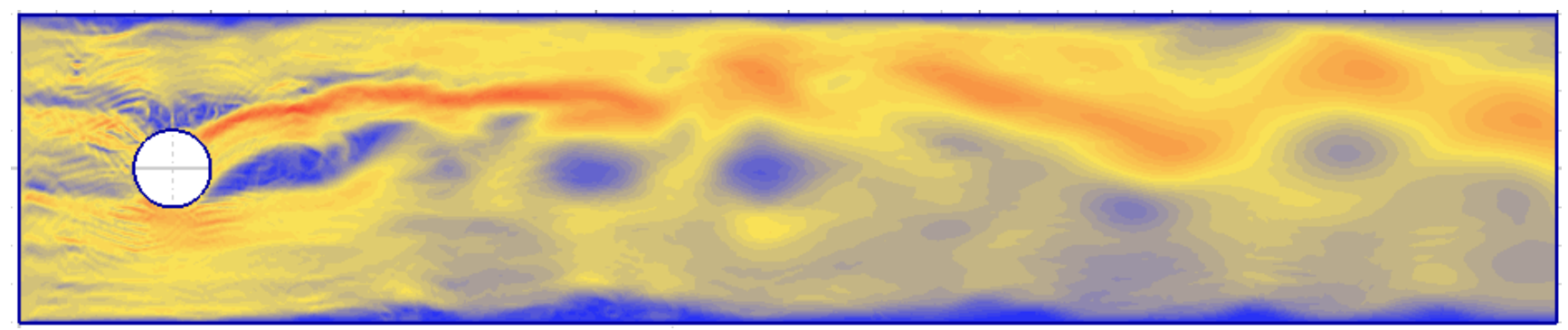

Fig. 6 Velocity map of a simulation of Magnus effect

of velocity at the lower part of the cylinder and increase of velocity at the lower surface of the cylinder is visible. The results shown correspond with the nature of the Magnus Effect, such as shown in Fig. 1.

Analysis of resource consumption was performed to assess Agros' load on the Graphics Processing Unit (GPU), Central Processing Unit (CPU), and RandomAccess Memory (RAM). Properties of the PC are presented in Table 2.

Overclocking software was used to monitor data usage. The analysis shown that the average GPU consumption did not increase. The RAM consumption raised from $4000 \mathrm{Mb}$ to $4350 \mathrm{Mb}$ for the duration of the experiment. Meanwhile, the CPU consumption had shown the highest growth. The results of CPU usage monitoring are presented in Fig. 7. After the simulation launch, the CPU usage has grown from $8 \%$ to a maximum of $73 \%$. Before the experiment, the average CPU usage was $4 \%$, and during the experiment, the

Table 2 Properties of the experimental PC.

\begin{tabular}{lc}
\hline Property & Value \\
\hline CPU & Intel(R) Core (TM) i7-6700HQ CPU @ $2.60 \mathrm{GHz}$ \\
GPU & NVIDIA GeForce GTX 1060 3Gb \\
RAM & $24.0 \mathrm{~Gb}$ \\
\hline
\end{tabular}

average CPU usage increased by $53 \%$ to an average of $57 \%$. The total simulation time was 94 minutes, which is comparable to other computational software.

\section{Conclusion}

The goal of presented work was to assess the possibility of using Agros for simulating and evaluating magnus effect. This approach has multiple advantages compared to traditional software used for CFD simulations. The main

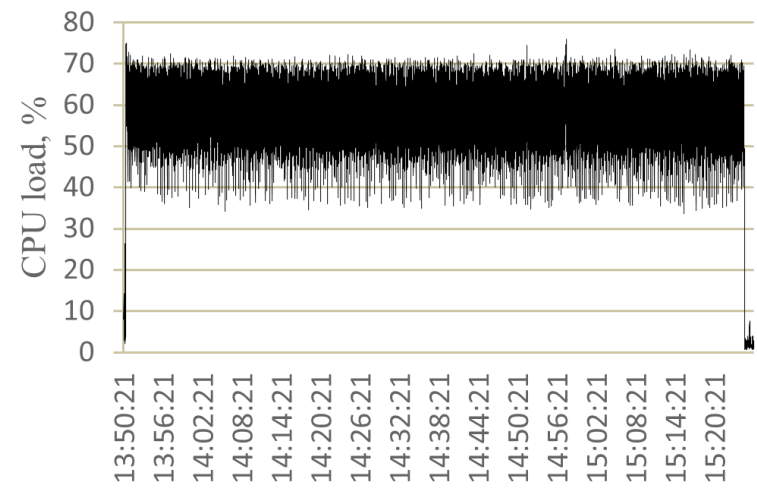

Simulation time

Fig. 7 Results of CPU load monitoring before, after, and during the simulation 
advantages of this software are its open software code, an ability to integrate Python scripts and built-in $h p$-adaptability, which positively affect simulation time and presents more opportunities for modifying source code and integrating the software into control systems. As a result of this work, a Magnus effect was achieved in open-source software package for solving physical problems - Agros 2D.

Agros 2D was shown to be a reliable tool in accessing and simulation the Magnus Effect. The application of this software allowed to study the fluid flow around a rotating body and observe dependencies between the rotational speed of the cylinder and flow separation pattern, and thus, Magnus lift. In the course of this work, two series of simulations were run to determine whether it is possible to observe and evaluate the Magnus Effect using the Agros software package. The difference in flow velocity around the upper and lower part of the cylinder was assessed in both simulations. Simulations have shown that with the increase of rotational frequency of the cylinder, the difference in velocities becomes more significant until it reaches the saturation point where the increase in rotational speed does not cause an increase in Magnus Effect force. The research has also shown that the margin of normal operational frequencies of the cylinder becomes wider at higher flow velocities. Thus, the simulation allowed to obtain data on saturation points of the experimental cylinder. A velocity map was also plotted, and visual analysis has shown that the Magnus Effect can be observed.

Monitoring software was ran in parallel with the simulation. Data logs from the software have shown that Agros does not apply significant load on video RAM (VRAM) and RAM usage. However, during the operation of the software, the CPU load increased significantly. Therefore, the research had shown high requirements for GPU proposed by Agros.

Further research was aimed at comparing Agros to similar CFD software. The research had shown similarities

\section{References}

[1] Lukin, A., Demidova, G. L., Lukichev, D. V., Rassõlkin, A., Kallaste, A., Vaimann, T., Belahcen, A. "Experimental Prototype of High-Efficiency Wind Turbine Based on Magnus Effect", In: 2020 27th International Workshop on Electric Drives: MPEI Department of Electric Drives 90th Anniversary (IWED), Moscow, Russia, 2020, pp. 1-6. https://doi.org/10.1109/IWED48848.2020.9069565

[2] Ericsson, L. E. "Moving wall effects in unsteady flow", Journal of Aircraft, 25(11), pp. 977-990, 1988.

https://doi.org/10.2514/3.45691

[3] Seifert, J. "A review of the Magnus effect in aeronautics", Progress in Aerospace Sciences, 55, pp. 17-45, 2012.

https://doi.org/10.1016/j.paerosci.2012.07.001 between results of simulation in Agros 2D and other FEM software. Despite discrepancies in numerical values, which can be explained by different solvers used in chosen software packages, simulations in in different CFD packages follow similar patterns, which was also confirmed by experimental data.

In conclusion, it can be said that Agros 2D was shown to be a reliable tool in accessing and simulation the Magnus Effect. Features of Agros, such as hp-adaptivity, opensource code, and Python scripting, provides competitive advantage in comparison to similar computational software. Further research in this field can include creating a framework for determining the lift force instead of the velocity map and integrating Agros 2D as a part of control system for the experimental wind generator to achieve optimal power control.

$\begin{array}{ll}\text { Nomenclature } \\ \text { MEWG } \\ \text { CPU } & \text { Central Processing Unit } \\ \text { GPU } & \text { Graphical Processing Unit } \\ \text { RAM } & \text { Random Access Memory } \\ \text { VRAM } & \text { Video Random Access Memory } \\ \text { CFD } & \text { Computational Fluid Dynamics } \\ L & \text { Lift force } \\ r & \text { Radius of the cylinder } \\ V & \text { Flow velocity } \\ G & \text { Strength of a Vortex } \\ \omega & \text { Angular velocity } \\ U & \text { Peripheral speed } \\ d & \text { Diameter of the cylinder } \\ n & \text { Rotational frequency } \\ U_{x} & \text { Projection of peripheral speed on X axis } \\ U_{y} & \text { Projection of peripheral speed on Y axis } \\ X_{0} & \text { Center of the cylinder on X axis } \\ x & \text { Location of a point on the surface of the cylinder }\end{array}$

[4] Lele, A., Rao, K. V. S. "Net power generated by flettner rotor for different values of wind speed and ship speed", In: 2017 International Conference on Circuit, Power and Computing Technologies (ICCPCT), Kollam, India, 2017, pp. 1-6. https://doi.org/10.1109/ICCPCT.2017.8074170

[5] Müller, M., Götting, M., Peetz, T., Vahs, M., Wings, E. "An Intelligent Assistance System for Controlling Wind-Assisted Ship Propulsion Systems", In: 2019 IEEE 17th International Conference on Industrial Informatics (INDIN), Helsinki, Finland, 2019, pp. 795-802. https://doi.org/10.1109/INDIN41052.2019.8972271 
[6] Lebkowski, A. "The concept of autonomous coastal transport", In: The International Conference on Marine Navigation and Safety of Sea Transportation (TRANSNAV 2017), Gdynia, Poland, 2017, pp. 351-357.

https://doi.org/10.1201/9781315099132-61

[7] Sedaghat, A. "Magnus type wind turbines: Prospectus and challenges in design and modelling", Renewable Energy, 62, pp. 619-628, 2014. https://doi.org/10.1016/j.renene.2013.08.029

[8] Luo, D., Huang, D., Wu, G. "Analytical solution on Magnus wind turbine power performance based on the blade element momentum theory", Journal of Renewable and Sustainable Energy, 3(3), Article Number: 033104, 2011 https://doi.org/10.1063/1.3588039

[9] Lukin, A., Rassõlkin, A., Demidova, G. L. "Estimation of International Standards for Unconventional Wind Turbine Testing", In: 2020 55th International Universities Power Engineering Conference (UPEC), Turin, Italy, 2020, pp. 1-6. https://doi.org/10.1109/UPEC49904.2020.9209890

[10] Argos Suite "Agros Suite: A multiplatform application for the solution of physical problems", [online] Available at: http://www. agros2d.org/ [Accessed: 16 February 2021]

[11] Pánek, D., Orosz, T., Karban, P. "Artap: Robust Design Optimization Framework for Engineering Applications", In: 2019 Third International Conference on Intelligent Computing in Data Sciences (ICDS), Marrakech, Morocco, 2019, pp. 1-6. https://doi.org/10.1109/ICDS47004.2019.8942318

[12] Karban, P., Pánek, D., Orosz, T., Petrášová, I., Doležel, I. "FEM based robust design optimization with Agros and Ārtap", Computers \& Mathematics with Applications, 81, pp. 618-633, 2021.

https://doi.org/10.1016/j.camwa.2020.02.010
[13] Andriushchenko, E., Kaska, J., Kallaste, A., Belahcen, A., Vaimann, T., Rassõlkin, A. "Design Optimization of Permanent Magnet Clutch with Ārtap Framework", Periodica Polytechnica Electrical Engineering and Computer Science, in press. (Accepted for publication: 14 September 2020)

[14] Orosz, T. "Evolution and Modern Approaches of the Power Transformer Cost Optimization Methods", Periodica Polytechnica Electrical Engineering and Computer Science, 63(1), pp. 37-50, 2019.

https://doi.org/10.3311/PPee.13000

[15] Mitchell, W. F., McClain, M. A. "A Comparison of hp-Adaptive Strategies for Elliptic Partial Differential Equations", ACM Transactions on Mathematical Software, 41(1), pp. 1-39, 2014. https://doi.org/10.1145/2629459

[16] Kiss, G. M., Kaska, J., de Oliveira, R. A. H., Rubanenko, O., Tóth, B. "Performance Analysis of FEM Solvers on Practical Electromagnetic Problems", [cs.CE], arXiv:2009.04399, Cornell University, Ithaca, NY, USA, 2020. [online] Available at: https:// arxiv.org/abs/2009.04399v1 [Accessed: 16 February 2021]

[17] Mara, B. K., Mercado, B. C., Mercado, L. A., Pascual, J. M., Lopez, N. S. "Development and validation of a CFD model using ANSYS CFX for aerodynamics simulation of Magnus wind rotor blades", In: 2014 International Conference on Humanoid, Nanotechnology, Information Technology, Communication and Control, Environment and Management (HNICEM), Palawan, Philippines, 2014, pp. 1-6. https://doi.org/10.1109/HNICEM.2014.7016231

[18] Sun, X., Zhuang, Y., Cao, Y., Huang, D., Wu, G. "A three-dimensional numerical study of the Magnus wind turbine with different blade shapes", Journal of Renewable and Sustainable Energy, 4(6), Article Number: 063139, 2012.

https://doi.org/10.1063/1.4771885 